\title{
Successful Management Of Cardiac Electrical Storm In Wellens Syndrome: A Case Report
}

Quoc Bao Tran ${ }^{1}$, Anh Binh Ho ${ }^{1}$, Van Duy Le ${ }^{1}$, Anh Khoa Phan ${ }^{1}$, and Van Khanh Vo ${ }^{1}$

${ }^{1}$ Hue Central Hospital

July 11, 2022

\begin{abstract}
A 42-year-old male patient diagnosed with Wellens syndrome, the angiography showed the narrowing not only in left anterior descending artery but also in other arteries. Cardiac electrical storm can occur without complete revascularization and it can be managed successfully with the infusion of amiodarone and lidocaine in different veins.
\end{abstract}

\section{Successful Management Of Cardiac Electrical Storm In Wellens Syndrome: A Case Report}

Anh Binh Ho ${ }^{1}$, Quoc Bao Tran ${ }^{*}$, Van Duy Le ${ }^{1}$, Anh Khoa Phan ${ }^{1}$, Van Khanh Vo ${ }^{1}$

\section{Cardiovascular emergency and intervention Department, Hue Central Hospital, Vietnam}

Corresponding author:

Quoc Bao Tran, Cardiovascular emergency and intervention Department, Hue Central Hospital, Vietnam, Tel: +84767054449

Email: quocbaotran29101995@gmail.com

\section{Successful Management Of Cardiac Electrical Storm In Wellens Syndrome: A Case Report}

\section{ABSTRACT}

A 42-year-old male patient diagnosed with Wellens syndrome, the angiography showed the narrowing not only in the left anterior descending artery but also in other arteries. Cardiac electrical storm can occur without complete revascularization and it could be managed successfully with the infusion of amiodarone and lidocaine along with cardiac defibrillation.

Keywords : Wellens syndrome, cardiac electrical storm, complete revascularization, infusion of amiodarone and lidocaine. 


\section{INTRODUCTION}

Wellens syndrome, also called left ascending artery (LAD) coronary syndrome or widow maker, was first described by de Zwaan et al in patients with unstable angina during a pain-free period with electrocardiographic (ECG) changes. ${ }^{1}$ There are two types of the syndrome, including type A and type B manifesting with biphasic T waves and deeply inverted T waves in leads V2-V3, respectively. ${ }^{2}$ If the patients present with these types of ECG, it is highly specific for severe, proximal stenosis of the left anterior descending coronary artery. ${ }^{3}$ Normally, when Wellens syndrome patients come to the emergency department or cardiovascular center, they do not have chest pain with normal or slightly elevated cardiac enzymes. ${ }^{4}$ However, it is crucial to attach the importance of the ECG patterns because these patients are at high risk for acute myocardial infarction with a large acute anterior wall. Percutaneous coronary intervention (PCI) is the definitive treatment to relieve the occlusion in LAD. ${ }^{5,6}$

The definition of cardiac electrical storm (ES) is three or more episodes of ventricular tachycardia (VT) or ventricular fibrillation (VF) in any interval of 24 hours. $^{7}$ There are many causes of ES, which can be divided into two categories scar-mediated re-entry due to previous myocardial infarction and reversible causes (acute ischemia, acute decompensated heart failure, electrolyte abnormalities, drug toxicity, sepsis and thyrotoxicosis). ${ }^{8}$ Electrical storm is considered a critical and severe situation both on management of hemodynamically unstable arrhythmias and its association with a significantly elevated sympathetic tone, which are likely to trigger further arrhythmias. ${ }^{9}$ Normally, patients with ES are treated with antiarrhythmic medications serially and suffer repeatedly from electrical shocks. ${ }^{10}$ The key intervention in the electrical storm is to revascularize if patients experiencing myocardial infarction and to block the sympathetic system through beta-blockers, especially propranolol; combined with analgesics and sedatives along with the control of serum electrolytes (particularly $\mathrm{Mg} 2+, \mathrm{K}+$ ) at high levels by the infusion of intravenous electrolyte solution. ${ }^{7,11-13}$

We would like to present a case of Wellens syndrome suffering from the cardiac electrical storm and how we successfully managed the patient.

\section{CASE REPORT}

A 42-year-old male patient with a history of type 2 diabetes under the treatment of $2000 \mathrm{mg} /$ day of Metformin presented with typical angina chest pain and dyspnea for 3 days before being hospitalized. The ECG findings showed the pattern of type A Wellens Syndrome (Figure 1), the echocardiography indicated the ejection flow reduced at $28 \%$ with contractile dysfunction in the wall motions of the left ventricular. The cardiac enzyme hs-Troponin $\mathrm{T}$ was $1.00 \mathrm{ng} / \mathrm{ml}$.

The patient was treated with enoxaparin $1 \mathrm{mg} / \mathrm{kg}$ q12hr, DAPT ( $81 \mathrm{mg}$ Aspirin and $75 \mathrm{mg}$ Clopidogrel), 40mg Rosuvastatin, 5mg Perindopril, 25mg spironolactone, low-dose of dobutamine $(5 \mathrm{mcg} / \mathrm{kg} / \mathrm{min}$ ) and insulin human (rDNA). The primary percutaneous coronary intervention (PCI) was performed for the patient with the angiographic result of severe and diffuse stenosis of 90-95\% in proximal LAD and significant stenosis of $80-90 \%$ in proximal LCx and proximal MR (Figure 2). We decided to deploy a drug-eluting stent in the LAD based on the ECG findings of Wellens syndrome (Figure 2).

The patient was hemodynamically stable after that. On day two after PCI, the patient suddenly suffered from cardiac arrest and was treated successfully with cardiopulmonary resuscitation, three times of defibrillation, and IV adrenaline. The ECG after 30 minutes showed premature ventricular contractions (PVC) bigeminy (Figure 3), it was managed by amiodarone with $150 \mathrm{mg}$ IV bolus and the IV maintenance dose of $1 \mathrm{mg} / \mathrm{min}$ for 6 hours and $0.5 \mathrm{mg} / \mathrm{min}$ IV after that. By doing so, the ECG finding was sinus rhythm with occasional PVCs. At that time, the serum potassium and magnesium were $3.8 \mathrm{mmol} / \mathrm{L}$ and $1.1 \mathrm{mmol} / \mathrm{L}$, respectively, which was under the IV infusion of electrolytes including 2-gram kali chloride $10 \%$ and 1.5-gram magnesium sulfate. Because of signs of congestive heart failure including rales at both lung bases, we were afraid of indicating beta-blockers for the patient.

The next day, while the patient was under treatment for a maintenance dose of amiodarone and electrolytes 
adjustment, he was still suffering from more than 5 times ventricular tachycardias (Figure 4) and intermittent ventricular fibrillation, which is also called cardiac electrical storm. The patient was managed with the combination of CPR and IV epinephrine, a number of defibrillations, 10mg diazepam for sedative and 1gram acetaminophen infusion of analgesics, IV potassium and magnesium, especially with the addition of one more antiarrhythmic drug called lidocaine with $1 \mathrm{mg} / \mathrm{kg}$ for the bolus dose and $1 \mathrm{mg} / \mathrm{min}$ for continuous infusion dose in different veins. After 30 minutes, the patient was stable hemodynamically with the sinus rhythm in ECG.

After that, we decided to transfer the patient to our cath-lab in order to perform PCI with drug-eluting stents in LCx and MR arteries (Figure 5). Thanks to the complete revascularization, the patient was stable with sinus rhythm.

The patient was followed up within a week and was discharged thanks to the hemodynamic stable condition with sinus rhythm and no arrhythmias. However, the cardiac fraction ejection did not improve. Therefore, the patient was treated with DAPT (81 $\mathrm{mg}$ Aspirin and $75 \mathrm{mg}$ Clopidogrel), high-dose of rosuvastatin at $40 \mathrm{mg}, 5 \mathrm{mg}$ of perindopril, 25mg of spinorolactone, low-dose of bisoprolol at 1.25mg, $2000 \mathrm{mg}$ of Metformin and $10 \mathrm{mg}$ of dapagliflozin. After 3 months of following up, the patient was stable with sinus rhythm and the cardiac function was improved from $28 \%$ to $48 \%$, which also meant we did not indicate implantable cardioverter-defibrillator for the prevention of sudden cardiac death due to ventricular fibrillation and ventricular tachycardia.

\section{DISCUSSION}

In our case, the patient with typical ECG of Wellens syndrome unluckily had severe and diffuse stenosis of $95 \%$ proximal LAD and significant stenosis of $80-90 \%$ proximal $\mathrm{LCx}$ and $80 \%$ proximal MR. The treatment included DAPT, enoxaparin, angiotensin-converting enzyme inhibitors, dobutamine, and insulin human (rDNA) along with primary PCI for the culprit lesion in LAD according to the ECG of Wellens syndrome, the patient was being followed up at the cardiac intensive care unit room. On the second day after PCI, the cardiac arrest occurred spontaneously due to ventricular tachycardia which was successfully managed by cardiac defibrillations and amiodarone. Despite that, in the next day, the episodes of VTs continued to occur and the patient was diagnosed with cardiac ES which was controlled by the addition of another antiarrhythmic drug called lidocaine with $1 \mathrm{mg} / \mathrm{kg}$ for the bolus dose and $1 \mathrm{mg} / \mathrm{min}$ for continuous infusion dose in different vein, along with the continuous dose of amiodarone at $0.5 \mathrm{mg} / \mathrm{min}$. The ECG was back with sinus rhythm and the patient was stable hemodynamically and immediately transferred to the catheterization room in order to perform PCI for the LCx and the MR. After PCI for LCx and MR artery, the patient did not show any arrhythmias in the ECG and the clinical examination was progressively better without chest pain and shortness of breath. However, the ejection fraction did not show any improvement. He was treated with DAPT (81 mg Aspirin and $75 \mathrm{mg}$ Clopidogrel), high-dose of Rosuvastatin at 40mg, 5 $\mathrm{mg}$ of Perindopril, and 25mg of Spinorolactone, low-dose of Bisoprolol at 1.25mg, $2000 \mathrm{mg}$ of Metformin and $10 \mathrm{mg}$ of Dapagliflozin. We intended to perform an Implantable Cardioverter-defibrillator (ICD) to prevent sudden cardiac death. However, after 3 months of following up on the above treatment, the patient was stable with sinus rhythm and the cardiac function was improved to $48 \%$, therefore, we decided not to do so and keep following up with the patient with the treatment.

Herein according to the joint EHRA, ACCA, and EAPCI task force in 2014 and 2017 AHA/ACC/HRS guidelines for management of patients with ventricular arrhythmias and the prevention of sudden cardiac death, patients with the acute coronary syndrome, presenting with the condition of incomplete revascularization as mentioned in our case with the significant stenosis of LCx and MR besides the culprit lesion in LAD, are at increased risk for the development of arrhythmia. ${ }^{16,17}$ Currently, there has been a lack of larger trials comparing the benefit from complete revascularization and incomplete revascularization. Generally, it is recommended that non-culprit lesion revascularization should be performed by a staged percutaneous coronary intervention in order to achieve complete revascularization. Therefore, after the successful PCI of the culprit lesion, it is advisable to treat other lesions in the duration of hospitalization. ${ }^{18}$ 
In addition, we believe that if one antiarrhythmic drug could not control the rhythm, the additional one in a different group should be considered. We suppose that intravenous infusion of amiodarone and lidocaine in different veins should be considered as an option for controlling ventricular arrhythmias along with the control of electrolytes, analgesics, and sedatives.

\section{CONCLUSIONS}

In conclusion, the Wellens syndrome patients suffering from cardiac electrical storm could be managed successfully if complete revascularization could be carried out. Additional, we would like to propose the intravenous infusion of amiodarone and lidocaine in different veins as an option for controlling the cardiac electrical storm along with cardiac defibrillation.

\section{List of abbreviations:}

CPR: cardiopulmonary resuscitation; DAPT: dual antiplatelet therapy; ECG: electrocardiography; ES: electrical storm; IV: intravenous; LAD: left anterior descending; LCx: left circumflex artery; MR: median ramus; PCI: percutaneous coronary intervention; PVC: premature ventricular contractions; VF: ventricular fibrillation; VT: ventricular tachycardia

\section{Consent for Publication}

For the publication of this case report, written informed consent was obtained from the patient.

\section{Disclosure}

The authors report no other conflicts of interest in this work.

\section{Acknowledgements}

I would like to show my gratitude to my dear teacher, Anh Binh $\mathrm{Ho}, \mathrm{PhD}$, who guided me the right way to manage my patient and helped me to make my decision to write my case report.

\section{REFERENCES}

1. de Zwaan C, Bär FW, Wellens HJ. Characteristic electrocardiographic pattern indicating a critical stenosis high in left anterior descending coronary artery in patients admitted because of impending myocardial infarction. American heart journal. 1982;103(4 Pt 2):730-6.

2. Miner B, Grigg WS, Hart EH. Wellens Syndrome. StatPearls. Treasure Island (FL)2021.

3. Mead NE, O'Keefe KP. Wellen's syndrome: An ominous EKG pattern. Journal of emergencies, trauma, and shock. 2009;2(3):206-8.

4. Lee Y, Habibzadeh MR, Movahed MR. Wellens Syndrome or Inverted U Waves: A Serious Clinical Condition Needing Immediate Attention Regardless of Symptoms. The American journal of medicine. 2021.

5. de Zwaan C, Bär FW, Janssen JH, Cheriex EC, Dassen WR, Brugada P, et al. Angiographic and clinical characteristics of patients with unstable angina showing an ECG pattern indicating critical narrowing of the proximal LAD coronary artery. American heart journal. 1989;117(3):657-65.

6. Rhinehardt J, Brady WJ, Perron AD, Mattu A. Electrocardiographic manifestations of Wellens' syndrome. The American journal of emergency medicine. 2002;20(7):638-43.

7. Conti S, Pala S, Biagioli V, Del Giorno G, Zucchetti M, Russo E, et al. Electrical storm: A clinical and electrophysiological overview. World journal of cardiology. 2015;7(9):555-61.

8. Tung R, Shivkumar K. Neuraxial modulation for treatment of VT storm. Journal of biomedical research. 2015;29(1):56-60.

9. Sagone A. Electrical Storm: Incidence, Prognosis and Therapy. Journal of atrial fibrillation. 2015;8(4):1150. 
10. Eifling M, Razavi M, Massumi A. The evaluation and management of electrical storm. Texas Heart Institute journal. 2011;38(2):111-21.

11. Collet J-P. 2020 ESC Guidelines for the management of acute coronary syndromes in patients presenting without persistent ST-segment elevation: The Task Force for the management of acute coronary syndromes in patients presenting without persistent ST-segment elevation of the European Society of Cardiology (ESC). European Heart Journal. 2021;42(14):1289-367.

12. Sorajja D, Munger TM, Shen WK. Optimal antiarrhythmic drug therapy for electrical storm. Journal of biomedical research. 2015;29(1):20-34.

13. Thomas DE, Jex N, Thornley AR. Ventricular arrhythmias in acute coronary syndromes - mechanisms and management. 2017;3(1):22-9.

14. Gupta S, Pressman GS, Figueredo VM. Incidence of, predictors for, and mortality associated with malignant ventricular arrhythmias in non-ST elevation myocardial infarction patients. Coronary artery disease. 2010;21(8):460-5.

15. Mehta RH, Starr AZ, Lopes RD, Hochman JS, Widimsky P, Pieper KS, et al. Incidence of and outcomes associated with ventricular tachycardia or fibrillation in patients undergoing primary percutaneous coronary intervention. Jama. 2009;301(17):1779-89.

16. Al-Khatib SM, Stevenson WG, Ackerman MJ, Bryant WJ, Callans DJ, Curtis AB, et al. 2017 AHA/ACC/HRS Guideline for Management of Patients With Ventricular Arrhythmias and the Prevention of Sudden Cardiac Death. 2018;138(13):e272-e391.

17. Gorenek B, Blomström Lundqvist C, Brugada Terradellas J, Camm AJ, Hindricks G, Huber K, et al. Cardiac arrhythmias in acute coronary syndromes: position paper from the joint EHRA, ACCA, and EAPCI task force. EuroIntervention : journal of EuroPCR in collaboration with the Working Group on Interventional Cardiology of the European Society of Cardiology. 2015;10(9):1095-108.

18. Thim T, van der Hoeven NW, Musto C, Nijveldt R, Gotberg M, Engstrom T, et al. Evaluation and Management of Nonculprit Lesions in STEMI. JACC Cardiovascular interventions. 2020;13(10):1145-54.

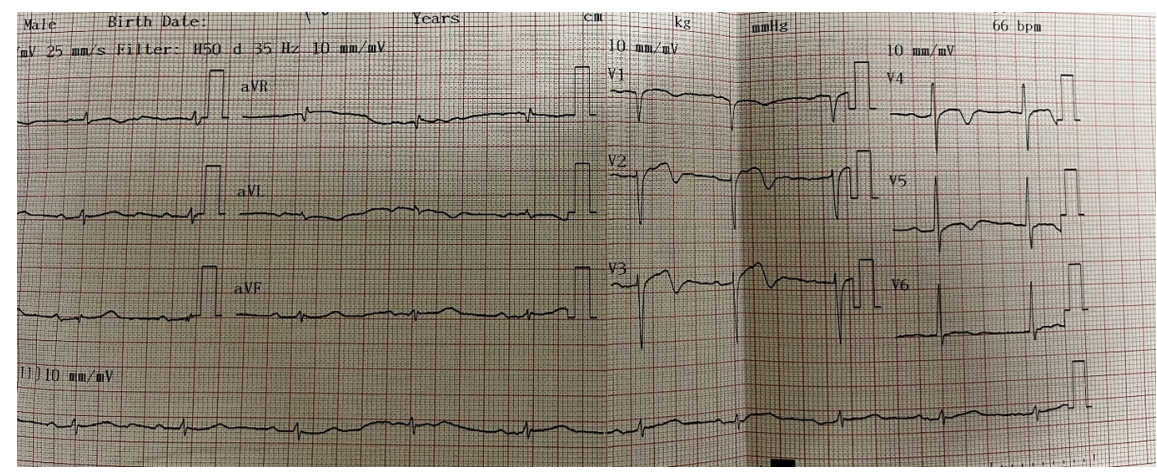

Figure 1. ECG findings on admission: Biphasic T waves in leads V2-V3 

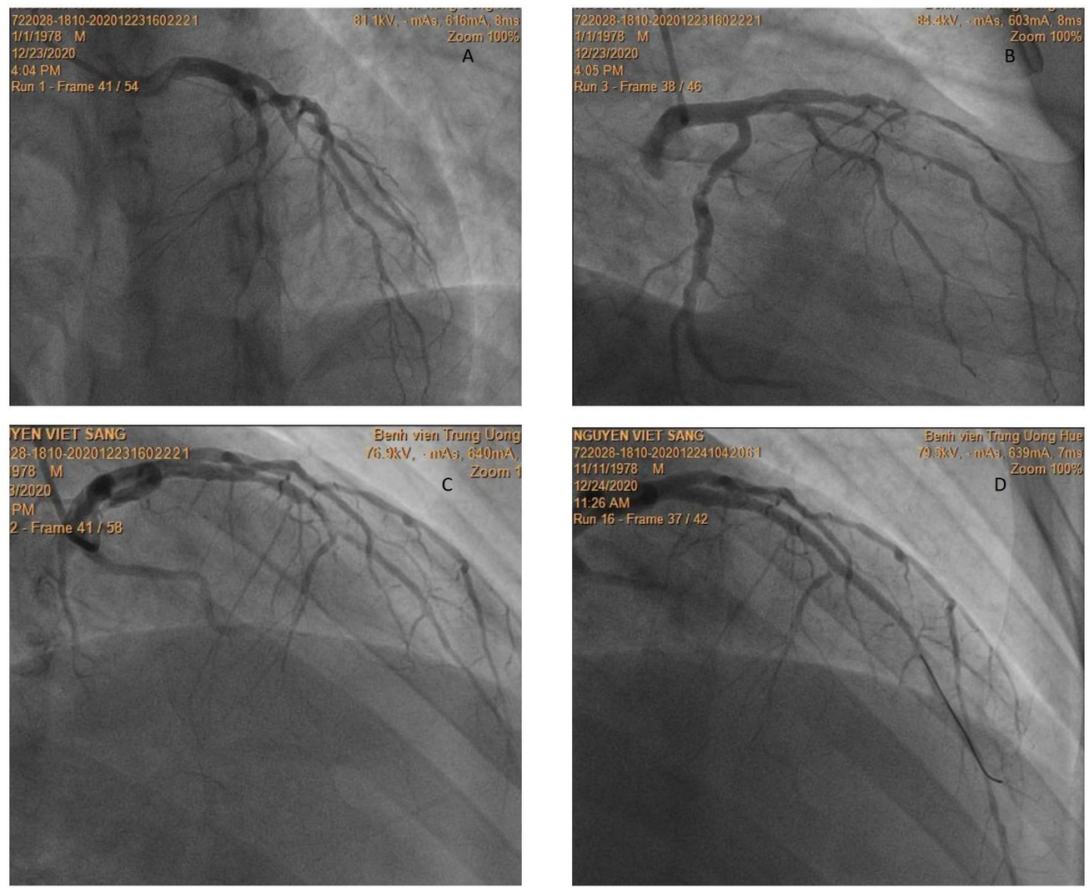

Figure 2. Coronary angiograms demonstrated the severe and diffuse stenosis in the proximal left anterior descending (LAD) (A, B, C), the significant stenosis also in the proximal left circumflex artery (LCx) and median ramus (MR) (B, C). Deploying a drug-eluting stent in LAD as a culprit lesion (D). 


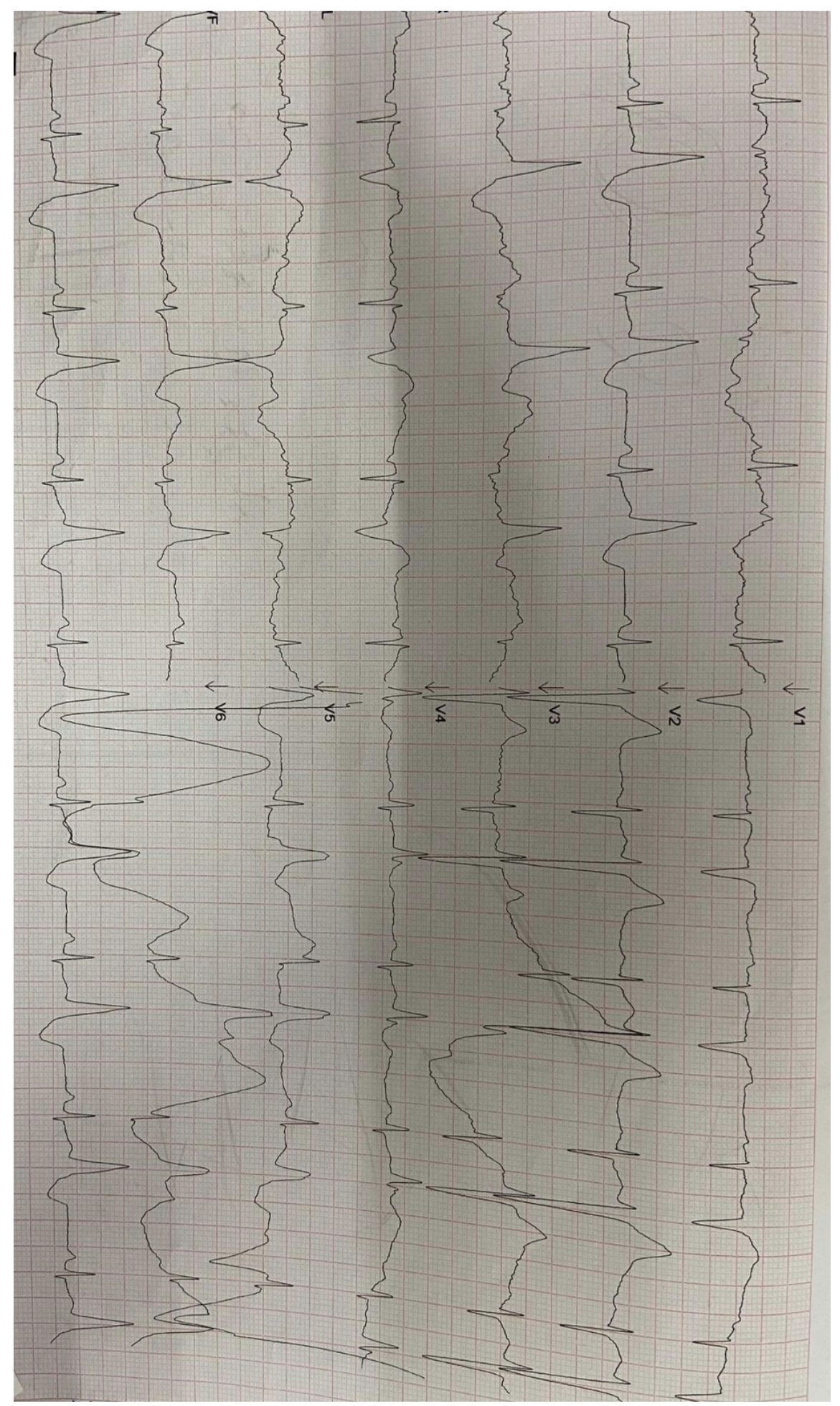

Figure 3. PVCs Bigeminy after successful management of sudden cardiac arrest 


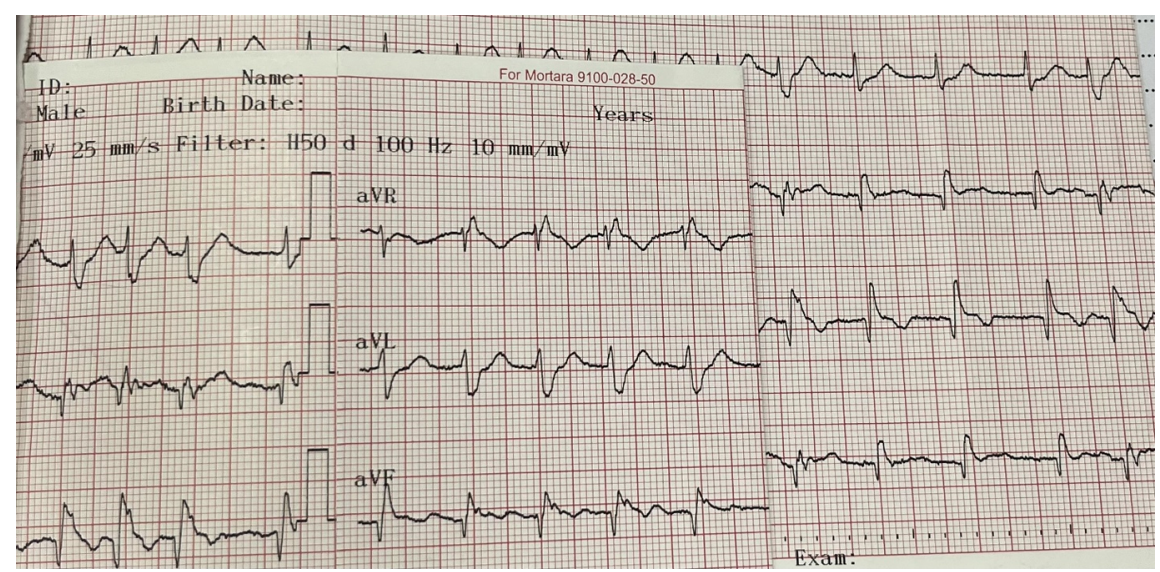

Figure 4. Monomorphic ventricular from the onset of electrical storm
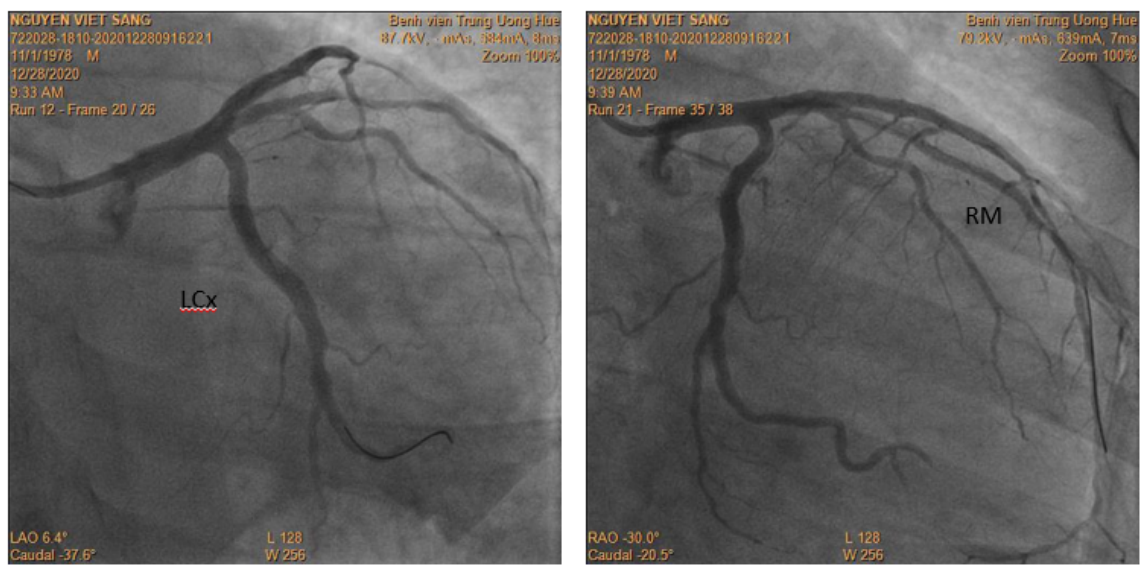

Figure 5. The final coronary angiograms after deploying percutaneous coronary interventions in LCx and RM with drug-eluting stents 